\title{
Red de coautoría de investigadores ambientalistas y su impacto en México
}

\author{
Co-Authorship Network of Environmental Researchers and Their Impact in \\ Mexico
}

\section{Rede de coautoria de pesquisadores ambientais e seu impacto no México}

Fernando Lámbarry Vilchis

Instituto Politécnico Nacional ESCA ST, Ciudad de México, México

flambarry@ipn.mx

https://orcid.org/0000-0002-0216-1647

\section{Resumen}

En México los investigadores más destacados son distinguidos por el Consejo Nacional de Ciencia y Tecnología. Aunque en la literatura internacional se han estudiado las redes de coautoría de los investigadores y el impacto de sus publicaciones, en el contexto mexicano este tipo de estudios son incipientes, por lo que el objetivo de este trabajo consistió en explorar la correlación entre dos métricas de centralidad y el índice $h$ de los investigadores ambientalistas eméritos, niveles dos y tres del país. Para ello, el método de investigación se fundamentó en la correlación de Pearson entre las métricas de centralidad de grado y de cercanía con el índice $h$. Se concluye que, a pesar de que los investigadores ambientalistas publican en forma colaborativa, por lo que promedian altos valores de centralidad de grado, no existe correlación con el impacto de sus publicaciones.

Palabras clave: impacto de las redes de coautoría, investigadores nacionales en ciencias ambientales, métricas de centralidad. 


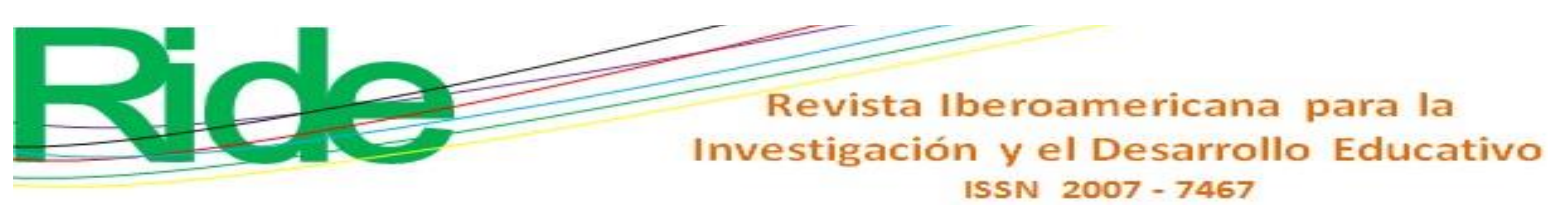

\section{Abstract}

In Mexico, the most outstanding researchers are distinguished by the Consejo Nacional de Ciencia y Tecnología. Although in the international literature researchers' co-authorship networks and the impact of their publications have been studied, in Mexico this type of studies is incipient, so the objective of this work is to explore the correlation between two centrality metrics and the $h$ index of environmental researchers emeritus, levels one and three of the country. For this, the research method was based on the Pearson correlation of the grade centrality and closeness metrics with the $h$ index. It is concluded that although environmental researchers publish in a collaborative way so they average high values of degree centrality, there is no correlation with the impact of their publications.

Keywords: impact of coauthorship networks, national research in environmental sciences, centrality metrics.

\section{Resumo}

No México, os pesquisadores mais proeminentes são distinguidos pelo Conselho Nacional de Ciência e Tecnologia. Embora as redes de coautoria de pesquisadores e o impacto de suas publicações tenham sido estudados na literatura internacional, no contexto mexicano esses tipos de estudos são incipientes, portanto o objetivo deste trabalho foi explorar a correlação entre duas métricas de centralidade e índice h de pesquisadores ambientais emeritus, níveis dois e três do país. Para tanto, o método de pesquisa baseou-se na correlação de Pearson entre as métricas de centralidade de grau e proximidade com o índice h. Conclui-se que, apesar do fato de os pesquisadores ambientais publicarem de forma colaborativa, portanto, medem os valores de centralidade de alto grau, não há correlação com o impacto de suas publicações.

Palavras-chave: impacto de redes de co-autores, pesquisadores nacionais em ciências ambientais, métricas de centralidade.

Fecha Recepción: Abril 2019

Fecha Aceptación: Agosto 2019 


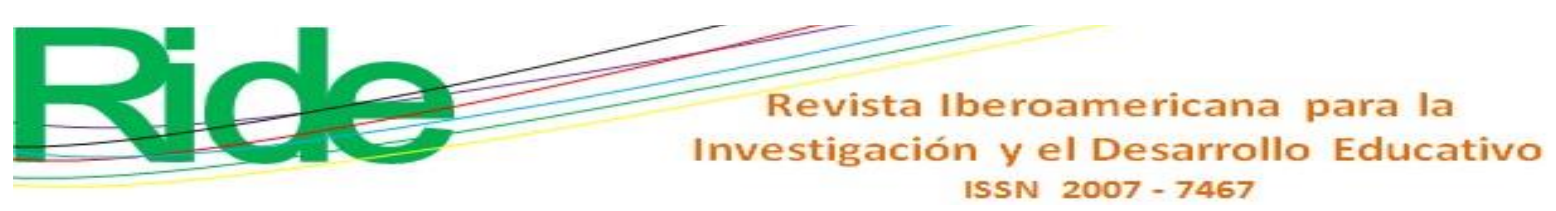

\section{Introducción}

En México, los investigadores en cualquiera que sea su disciplina de trabajo son reconocidos por el Sistema Nacional de Investigadores (SNI) en alguno de sus cinco niveles: candidato, nivel uno, nivel dos, nivel tres y emérito; estos últimos son de los más distinguidos por la calidad de sus publicaciones. Si bien los investigadores pueden exhibir diferentes tendencias en la colaboración, hay una creciente popularidad en publicar en coautoría independientemente de la disciplina de la que forman parte (Lopaciuk-Gonczaryk, 2016; De Stefano, Fuccella, Prosperina y Zaccarin, 2013; Kronegger, Ferligoj y Doreian, 2011), aunque en diversos grados, difiriendo de un país a otro, e incluso entre disciplinas (Liberman y Wolf, 2013; Yu Cheng, Wah Hen, Piew Tan y Fai Fok, 2013).

La coautoría es un producto explícito de la colaboración científica con patrones relacionales que han sido explicados por estructuras y propiedades topológicas de las redes fundamentadas en la teoría de la centralidad (Newman, 2001a, 2001b, 2004a; Barabasi y Albert, 1999; Freeman, 1979); además, posibilitan respuestas a preguntas tales como "quién es el autor más central de la red" y "si existe una relación entre la colaboración y la productividad del autor" (Kumar y Mohd, 2014, p. 356). Al respecto, estudios anteriores han evidenciado que la colaboración en investigación produce un mayor impacto que un solo investigador en términos del número de publicaciones (Lotka, 1926; Katz y Hicks, 1997; Lee y Bozeman 2005; Ponomariov y Boardman, 2010; De Stefano et al., 2013) y citas (Gazni, Sugimoto y Didegah, 2012; Sooryamoorthy, 2009). También que las relaciones entre la colaboración y el impacto científico parecen ser más positivas en las ciencias duras, como la física y la astronomía, que en las ciencias blandas, como la sociología (Bridgstock, 1991) y la ecología (Peters, 1991).

Sorprendentemente, son pocos los estudios que han probado esta proposición, y particularmente los orientados al ámbito de las ciencias ambientales. Por lo que el objetivo de esta investigación consistió en explorar la naturaleza de la correlación entre la centralidad y el impacto de las publicaciones (índice $h$ ) de los investigadores nacionales ambientalistas eméritos, niveles dos y tres de México. Para lograrlo, el método de investigación se fundamentó en las métricas de centralidad de Freeman y de correlación de Pearson. 
Base teórica

\section{Impacto de la colaboración científica}

La publicación de los resultados de investigación es uno de los principales impulsores del prestigio de las instituciones por ser uno de los criterios en las clasificaciones que jerarquizan a las universidades de todo el mundo, en donde la publicación académica en revistas indexadas juega un papel importante en sus métodos de puntuación (Munoz, Queupil y Fraser, 2016). La posición de las universidades en el ranking puede tener un impacto directo tanto en su matriculación de estudiantes como en su financiamiento (Dill y Soo, 2005; Dill, 2009). Por lo que cuantificar el impacto científico de un autor se ha convertido en la métrica por excelencia para las facultades y/o centros de investigación.

En la bibliometría y en la cienciometría se utilizan diversas medidas del rendimiento de un investigador, tales como el recuento de artículos (Lee y Bozeman, 2005); el número de citas proporcionado por Clarivate, antes Web of Knowledge de ISI (McFadyen y Cannella, 2017; Badar, Hite y Yuosre, 2013); el índice de impacto Hirsch (h) (Pike, 2010; Sidiropoulos, Katsaros y Manolopoulos, 2007; Batista, Campitel, Kinouchi y Martinez, 2006; Kelly y Jennions, 2006; Hirsch, 2005); y el índice de Leo Egghe (G) (Costas y Bordons, 2007; Egghe, 2006; Van Raan, 2006) y el índice Jin BiHui (R). No obstante, todos ellos indican el grado en que el trabajo de un científico ha sido utilizado por otros investigadores (Bornmann, Mutz, Neuhaus y Daniel, 2008), lo que a su vez puede derivar en que aquellos investigadores que publican más en términos de factor de impacto atraen a más potenciales colaboradores, tienen un mayor rendimiento (Li, Liao y Yen, 2013; Liao, 2011) y se vuelven, desde la perspectiva de la teoría de la centralidad, en investigadores más centrales.

\section{Redes de coautoría científica: índice de impacto y métricas de centralidad}

Desde el estudio clásico de Zuckerman (1967), en el que analizó los patrones de investigación de 41 premios Nobel y evidenció empíricamente que los galardonados son muy selectivos al elegir a sus colaboradores pues seleccionan a científicos de renombre y productivos. Zuckerman (1967) identificó una fuerte relación entre la colaboración y la productividad. Estudios más recientes se han enfocado en el impacto científico de las publicaciones de los investigadores como una métrica de su productividad. Ejemplo de ello es el realizado por Pike (2010), quien, al emplear el índice de impacto $(h)$, determinó que los 


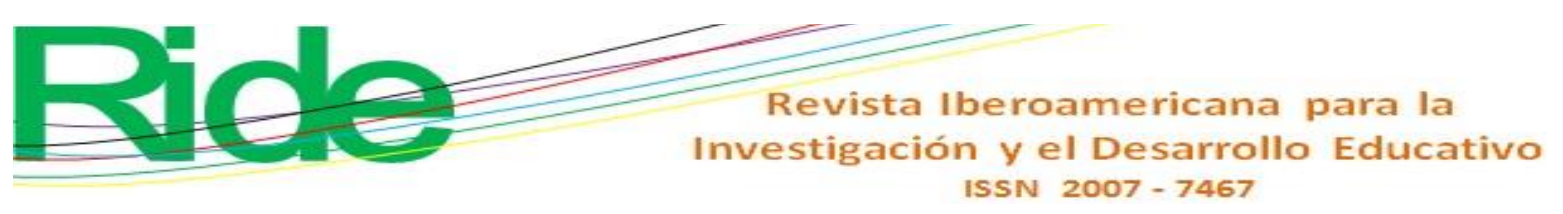

investigadores con altos índices $h$ tienden a colaborar con otros científicos de alto impacto, mientras que los que tienen un bajo índice de impacto parecen no desear colaborar con otros menos exitosos que ellos. En otros estudios se ha identificado la influencia positiva entre la centralidad de los investigadores y la eficiencia en las redes de coautoría: Badar, Hite y Badir (2014) en los científicos en química de Pakistán; Bordons, Aparicio, González-Albo y DíazFaes (2015) en los de nanociencia, farmacología y estadística de España; Eaton, Ward, Kumar y Reingen (1999) en los investigadores del comportamiento del consumidor; Fischbach, Putzke y Schoder (2011) en la investigación de mercados electrónicos; GonzálezBrambila (2014) en los científicos en ciencias sociales de México; Lee, Seo y Choe (2012) en campos de ciencia e ingeniería de las instituciones públicas de investigación de Corea.

Si bien los estudios antes mencionados han probado rigurosamente que la centralidad de la red conduce a la hipótesis de rendimiento - mientras que otros más demuestran que los grados y tipos de colaboración difieren de un país a otro y de disciplina a disciplina (Newman, 2004a, 2004b, Liberman y Wolf, 2013; Yu Cheng Wah Hen, Piew Tan y Fai Fok, 2013)—; el caso de los investigadores en las ciencias ambientales no ha sido analizado con la misma profundidad con la que lo ha sido para otras ciencias, aunque entre ellos se encuentra el de Pike (2010) para la ecología del comportamiento bajo un enfoque correlacional entre el coeficiente de agrupamiento y el impacto científico, y el de Kumar y Mohd (2014) para los científicos de la tierra en la India, en el que concluyeron con la existencia de una fuerte correlación entre la centralidad de grado e intermediación con la productividad del autor (número de trabajos).

\section{Método de investigación}

El objetivo de este estudio consistió en analizar la correlación entre la centralidad de cercanía y el impacto de las publicaciones (índice $h$ ) de los investigadores ambientalistas eméritos, niveles dos y tres de México. Para ello, la metodología de corte cuantitativo implicó dos fases: una primera de recopilación y preparación de la base de datos de las publicaciones y del índice $h$ de Scopus (impacto) de los investigadores bajo estudio; y una segunda fase de análisis cuantitativo para calcular las métricas de centralidad de cada uno de los investigadores y de análisis correlacional con el impacto de las mismas. A continuación se describen las fases más ampliamente: 


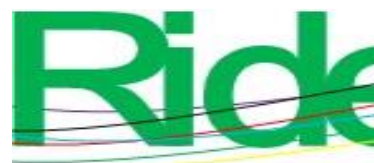

Revista Iberoamericana para la

Investigación y el Desarrollo Educativo ISSN $2007-7467$

\section{Fase de recopilación y preparación de la base de datos}

No existe en el Consejo Nacional de Ciencia y Tecnología (Conacyt) una categoría para los científicos en ciencias ambientales, por lo que identificar a los investigadores en este campo implicó seleccionar, a partir del listado de investigadores vigentes de enero a diciembre 2016, a los científicos de las disciplinas relacionadas con el estudio del medio ambiente. De esta forma, se identificaron siete campos disciplinares: climatología, desarrollo sustentable, ecología, medio ambiente, oceanografía, tecnología del medio ambiente y otras especialidades. A partir de ello, se reconocieron a los investigadores nacionales eméritos y niveles dos y tres de México de las ciencias del medio ambiente. En total resultaron 88 investigadores activos, de quienes, a través de una consulta pública realizada el 2 de enero de 2017 al Instituto Nacional de Transparencia, Acceso a la Información y Protección de Datos Personales (INAI), se adquirió y recopiló su productividad de artículos del período de 2012 al 2016.

En la preparación de la base de datos cada uno de los artículos fue verificado en las revistas donde fueron publicados y validados en cuanto a los coautores participantes. Con ello se creó una base de datos con un total de 3642 publicaciones, de las cuales 3537 corresponden a artículos en coautoría y 105 a autorías únicas (véase anexo tabla 3). Se identificaron en total a 4751 autores en las publicaciones, de los cuales 4663 son coautores y el restante investigadores que publican de forma individual. No obstante, en algunos de ellos implicó corregir la desambiguación de sus nombres, es decir, se verificaron los registros para visualizar las variaciones de los nombres de los autores, además de los caracteres incorrectos de la propia base de datos del Conacyt. La forma de desambiguar fue validando la afiliación institucional de los autores.

\section{Fase de análisis cuantitativo y correlacional}

Esta fase implicó un estadístico descriptivo de la productividad de los artículos de los investigadores. Además, para evaluar en forma individual el impacto de los investigadores, se seleccionó el índice $h$ de Scopus y se comparó entre los científicos de las diferentes disciplinas ambientales. Un investigador tiene un índice $h$ si $h$ de sus $N_{p}$ publicaciones tienen al menos $h$ citas cada uno, y el otro $\left(N_{p}-h\right)$ publicaciones tienen $\leq h$ citas cada uno (Hirsch, 2005). 


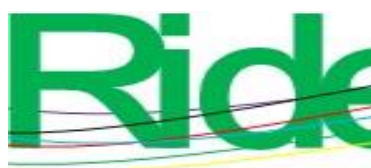

Revista Iberoamericana para la
Investigación y el Desarrollo Educativo
ISSN $2007-7467$

Para el análisis de la red de coautoría de los investigadores ambientalistas se calculó la centralidad para cada uno de ellos a partir de la base de datos de sus publicaciones. Se consideraron dos de las tres métricas clásicas de Freeman (1979), las cuales son comúnmente utilizadas y que son el objeto de interés de este estudio: analizar la comunicación (grado) y la independencia (cercanía), no así el control de la comunicación (intermediación).

La centralidad de grado $C_{D}$ de un nodo es la medida más simple e intuitiva sobre su actividad potencial de comunicación (Freeman, 1979); representa la cantidad de aristas que se le atribuyen, sin tener en cuenta la intensidad de la conexión. El grado de un nodo $p_{i}$ es simplemente el número de nodos $p_{j}(i \neq j)$ que son adyacentes a él (Nieminen, 1974). Se calcula como el grado o el número de adyacencias para el nodo $p_{k}: C_{D}\left(p_{k}\right)=\sum_{i=1}^{n} a\left(p_{i}, p_{k}\right)$.

La centralidad de cercanía $C^{\prime}{ }_{c}$ se basa en el grado en el que un nodo está cerca de los demás nodos de la red. Aquí un nodo se considera central en la medida en que puede evitar el potencial control de los otros. En realidad, se trata de una medida de descentralidad o de centralidad inversa, ya que crece a medida que los puntos se separan, y la centralidad en este contexto significa cercanía (Freeman, 1979). Matemáticamente es: $C_{c}^{\prime}\left(p_{k}\right)=\frac{n-1}{\sum_{i=1}^{n} d\left(p_{i}, p_{k}\right)}$, donde $d\left(p_{i}, p_{k}\right)$ es el número de aristas en la geodésica (distancia más corta de dos nodos) que une a $p_{i} y p_{k}$. Las métricas de centralidad fueron computadas mediante el programa Cytoscape.

Finalmente, para el cálculo del índice de asociación de las variables estudiadas, se utilizó el coeficiente de correlación de Pearson computado en SPSS.

\section{Resultados}

Se identificaron a 88 investigadores nacionales en las ciencias ambientales, todos ellos en siete diferentes campos disciplinares. De ellos, 65 se encuentran en el nivel dos, en el nivel tres están 22 y un solo emérito. La disciplina de tecnología del medio ambiente, con un total de 40, es la que cuenta con el mayor número de investigadores. Ecología es la disciplina que cuenta con un único investigador y es emérito (ver anexo). 


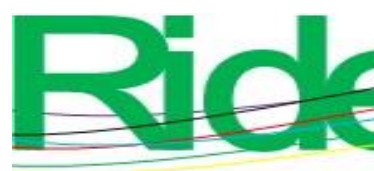

Revista Iberoamericana para la Investigación y el Desarrollo Educativo ISSN 2007 - 7467

\section{Descripción de la productividad de los investigadores}

Se distingue un importante volumen de publicaciones en coautoría con $97 \%$ de los investigadores ambientalistas, mientras que las realizadas por un autor individual representan el restante $3 \%$. Estos porcentajes son muy cercanos en seis de las siete disciplinas, salvo en ecología, donde el investigador más destacado de las ciencias ambientales publica todos sus artículos en colaboración con otros.

En tanto que desarrollo sustentable y medio ambiente muestran valores atípicos de hasta 49 coautores por publicación. La primera, a pesar de que cuenta con siete investigadores nacionales, conlleva una gran diversidad de coautores en sus publicaciones. Esto puede explicarse por alguna reciprocidad colaborativa a razón de ser la disciplina con la mayor cantidad de publicaciones, mientras que medio ambiente se posiciona en el tercer lugar. En este sentido, todas las disciplinas cuentan con artículos publicados en coautoría con 10 investigadores o más, a excepción de ecología que tiene máximo cinco y climatología hasta nueve. No obstante, para los dos últimos años (2015 y 1026) se aprecia una disminución en ambas formas de publicación, lo que es atribuible en gran parte a la falta de actualización del currículo (CVU) de Conacyt por parte de los investigadores (véase figura 1).

Figura 1. Número de publicaciones y coautores

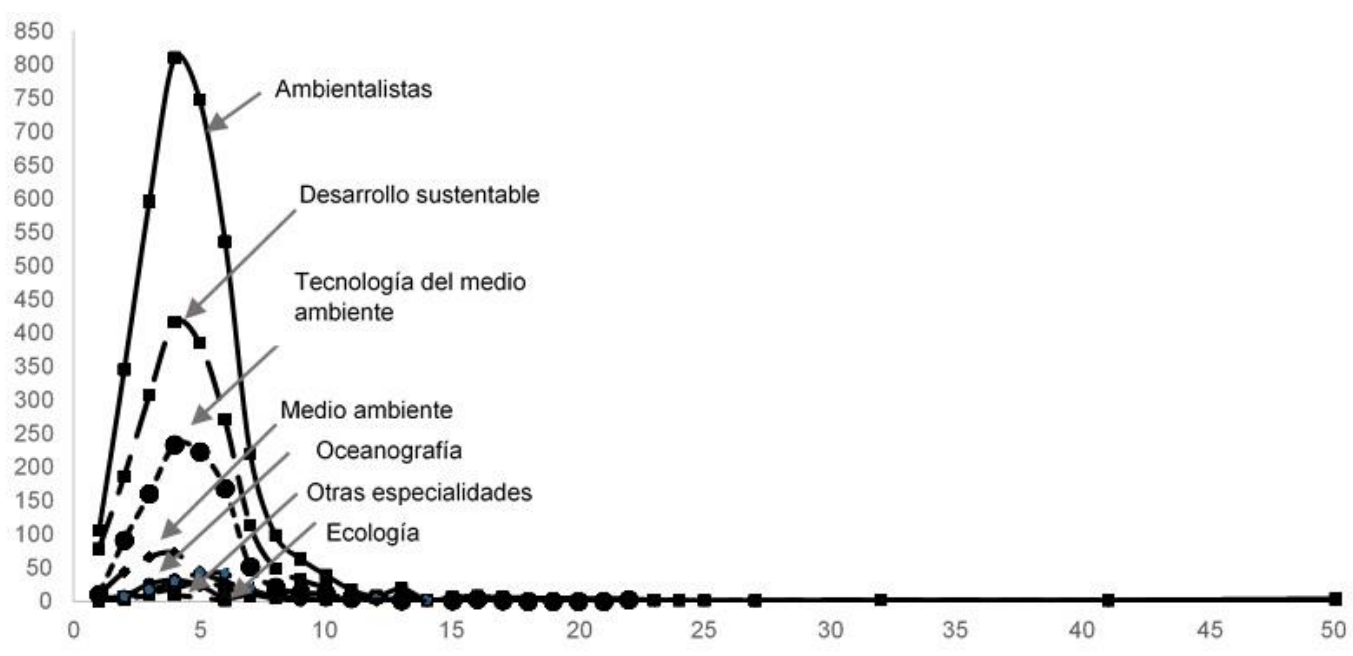

Fuente: Elaboración propia

En este mismo sentido, en promedio cada uno de los investigadores publica ocho artículos al año. Aunque desde la perspectiva particular por disciplina, se observa que el investigador emérito colabora en cinco publicaciones anuales, lo que contrasta con desarrollo 


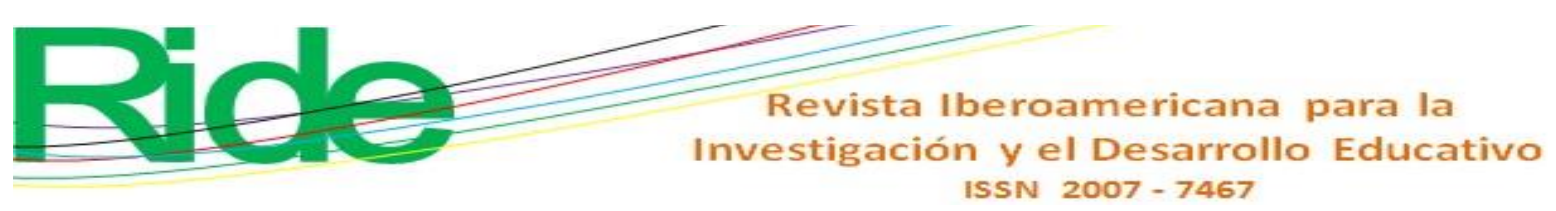

sustentable, que es una de las disciplinas con el menor número de investigadores nacionales con siete, solo por arriba de oceanografía, que tiene seis, y ecología, que cuenta con solamente uno. No obstante, desarrollo sustentable es el campo con más artículos publicados con un total de 1901, lo que representa $52 \%$ del total de las publicaciones de esta ciencia; de ellos, 1823 son en coautoría (representa $50 \%$ del total de los artículos escritos colaborativamente) versus 78 que se escriben en forma individual. Derivado de lo anterior, no es de extrañarse que desarrollo sustentable promedia el valor más alto de publicaciones por investigador nacional con cuatro durante el período analizado y la que cuenta con la mayor cantidad de coautores por artículo.

Por debajo del número de publicaciones de desarrollo sustentable está tecnología del medio ambiente, con 993, y es la disciplina que concentra al mayor número de investigadores con 40 , lo que representa $45 \%$ del total de los científicos y $27 \%$ del total de las publicaciones en coautoría, lo que la convierte en la segunda con la mayor cantidad de trabajo colaborativo de las ciencias ambientales. En un tercer lugar, se encuentra la disciplina de medio ambiente que, con $17 \%$ de investigadores nacionales de este campo, contribuye con $8 \%$ del total de las publicaciones en coautoría.

En este sentido, desarrollo sustentable es la única área ambientalista que contribuye de forma sensible respecto de la totalidad de los artículos publicados en forma individual con $3 \%$. De esta forma, se concluye que los investigadores ambientalistas publican en forma colaborativa.

\section{Correlación: centralidad de grado y de cercanía con el índice $h$}

Las métricas de centralidad revelan la concentración de autoridad, control u otros recursos dentro de la red. La tabla 1 sintetiza las características y las métricas de centralidad de la red de los investigadores nacionales de las ciencias ambientales, así como de las redes disciplinarias que la componen. 


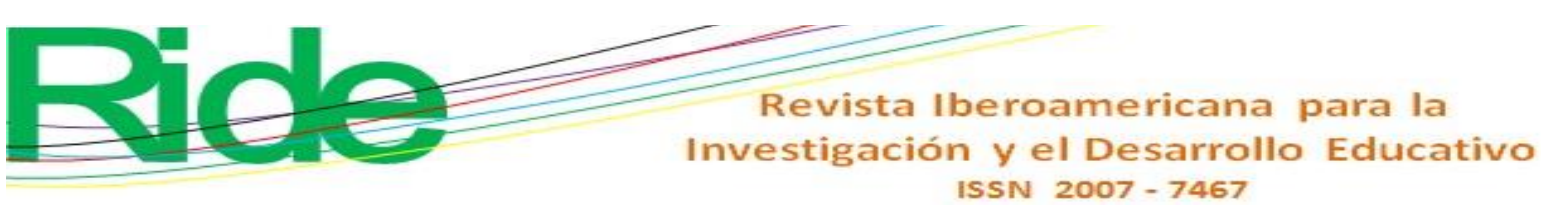

Tabla 1. Características y métricas de centralidad de las redes de coautoría ambientalistas

\begin{tabular}{|c|c|c|c|c|c|c|c|c|}
\hline Métricas/Áreas & Am & $\mathrm{Cl}$ & DS & Ec & MA & Oc & TMA & Otras \\
\hline \multicolumn{9}{|l|}{ Características } \\
\hline \multirow{3}{*}{ Total SNI } & 88 & \multirow{3}{*}{$\begin{array}{c}6 \\
(7 \%)\end{array}$} & \multirow{3}{*}{$\begin{array}{c}7 \\
(8 \%)\end{array}$} & \multirow{3}{*}{$\begin{array}{c}1 \\
(1 \%)\end{array}$} & 15 & 10 & 40 & 9 \\
\hline & $(100$ & & & & $(17$ & $(11$ & $(45$ & $(10$ \\
\hline & $\%)$ & & & & $\%)$ & $\%)$ & $\%)$ & $\%)$ \\
\hline Nivel 2 & 65 & 4 & 4 & 0 & 10 & 8 & 31 & 8 \\
\hline Nivel 3 & 22 & 2 & 3 & 0 & 5 & 2 & 9 & 1 \\
\hline Emérito & 1 & 0 & 0 & 1 & 0 & 0 & 0 & 0 \\
\hline $\begin{array}{l}\text { Número de } \\
\text { artículos }\end{array}$ & 3642 & 110 & 1901 & 22 & 315 & 119 & 993 & 182 \\
\hline Número de autores & 4751 & 297 & 278 & 32 & 1057 & 393 & 2110 & 584 \\
\hline \multirow{3}{*}{$\begin{array}{r}\text { Artículos autor } \\
\text { único }\end{array}$} & 105 & 4 & 78 & \multirow{3}{*}{$\begin{array}{c}0 \\
(0 \%)\end{array}$} & 10 & 1 & 10 & 2 \\
\hline & $(2.88$ & $(3.64$ & $(4.10$ & & $(3.17$ & $(0.84$ & (1.01 & $(1.10$ \\
\hline & $\%)$ & $\%)$ & $\%)$ & & $\%)$ & $\%)$ & $\%)$ & $\%)$ \\
\hline \multirow{3}{*}{$\begin{array}{r}\text { Artículos en } \\
\text { coautoría }\end{array}$} & 3537 & 106 & 1823 & 22 & 305 & 118 & 983 & 180 \\
\hline & $(97.12$ & (96.36 & $(95.90$ & $(100$ & (96.83 & (99.16 & (98.99 & (98.90 \\
\hline & $\%)$ & $\%)$ & $\%)$ & $\%)$ & $\%)$ & $\%)$ & $\%)$ & $\%)$ \\
\hline $\begin{array}{l}\text { Promedio de } \\
\text { autores por }\end{array}$ & 8.49 & 5 & 14.79 & 2.75 & 14.3 & 6.75 & 8.86 & 7 \\
\hline $\begin{array}{r}\text { Promedio de } \\
\text { artículos nor autor }\end{array}$ & 3.77 & 4.05 & 3.74 & 2.40 & 4.09 & 3.86 & 3.70 & 4.59 \\
\hline Grado promedio & 14.80 & 12.49 & 17.55 & 10.87 & 17.36 & 10.47 & 14.09 & 12.91 \\
\hline Cercanía promedio & 0.215 & 0.502 & 0.507 & 0.569 & 0.377 & 0.513 & 0.234 & 0.299 \\
\hline
\end{tabular}

Nota: Am: Ambientalistas, Cl: Climatología, DS: Desarrollo sustentable, Ec: Ecología, MA: Medio ambiente, Oc: Oceanografía, TMA: Tecnología del medio ambiente, Otras: Otras especialidad medioambientales.

Fuente: Elaboración propia 


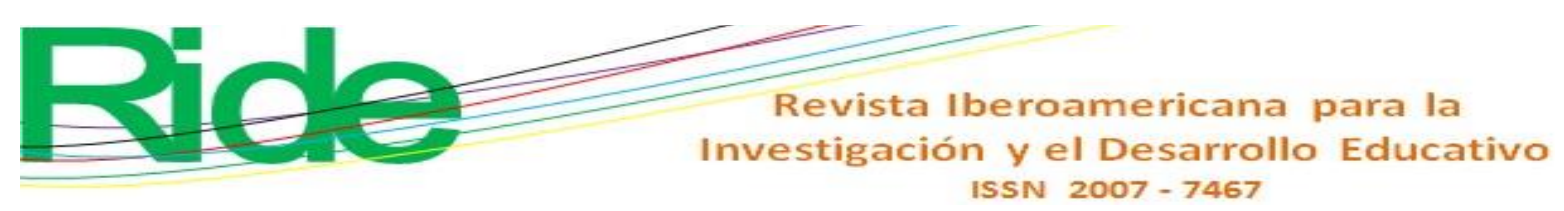

Discusión

\section{Centralidad de grado}

El grado promedio de autores en la red (componente gigante) presenta un valor de 14, lo que muestra que los autores en la red ambientalista, en general, están bien conectados. No obstante, desarrollo sustentable con 17 y medio ambiente también con 17 son las disciplinas con los valores promedios más altos de grado. En contraste, oceanografía tiene el grado promedio más bajo con 10. En este sentido, aquellos autores con grados más altos se convierten por su posición en los más influyentes.

\section{Centralidad de cercanía}

La centralidad de cercanía promedio de la red es de 0.215 , y asociados a 6 grados promedio de separación. Es ecología con 0.569 la disciplina con el mayor coeficiente de cercanía; climatología y desarrollo sustentable cuentan también con coeficientes por arriba de 0.5. Lo que indica que sus nodos se encuentran más cerca de los demás nodos de la red, por lo que en estas redes se encuentran los investigadores más centrales que pueden evitar el potencial control de los otros.

\section{Correlación entre las métricas de centralidad y el índice $\boldsymbol{h}$ de impacto}

Por una parte, el coeficiente de correlación entre la centralidad de grado y el índice $h$ (véase tabla 2), considerando en su conjunto a las disciplinas ambientalistas, es de un valor de 0.131 con una significancia bilateral de 0.223 , lo que indica una correlación demasiado débil pero no significativa. Para la métrica de cercanía y el índice $h$ (tabla 2), el coeficiente de correlación es de -0.107 y una significancia bilateral de 0.322 , por lo que presenta un grado de asociación mínimo y no significativo. De esta forma, se concluye que no existe correlación alguna entre la centralidad de grado y/o de cercanía con el índice de impacto de los científicos ambientalistas eméritos y niveles dos y tres de México. Sin embargo, los investigadores de otras especialidades ambientales tienen una correlación alta de $0.744 *$, significativa en el nivel 0.05 (dos colas) de su centralidad de grado con el impacto de sus obras publicadas. Es la única de las disciplinas que presenta algún valor en términos de esta asociación. 


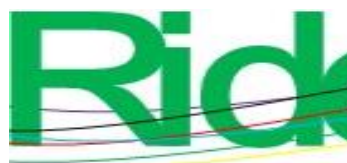

Revista Iberoamericana para la Investigación y el Desarrollo Educativo ISSN $2007-7467$

Tabla 2. Correlación por disciplina de los investigadores nacionales niveles dos y tres

\begin{tabular}{|c|c|c|c|c|c|c|}
\hline \multirow{3}{*}{ Disciplina } & \multicolumn{6}{|c|}{ Correlaciones de Pearson } \\
\hline & \multicolumn{3}{|c|}{ Grado vs. índice $h$} & \multicolumn{3}{|c|}{ Cercanía vs. índice $h$} \\
\hline & SNI's & Nivel 2 & Nivel 3 & SNI's & Nivel 2 & Nivel 3 \\
\hline \multirow{2}{*}{$\begin{array}{l}\text { Todas las } \\
\text { disciplinas }\end{array}$} & 0.131 & $0.322 * *$ & -0.068 & -0.107 & -0.009 & $-0.558 * *$ \\
\hline & $N=88$ & $N=65$ & $N=22$ & $N=88$ & $N=65$ & $N=22$ \\
\hline Ecología $^{1}$ & No aplica & $\begin{array}{l}\text { No } \\
\text { aplica }\end{array}$ & $\begin{array}{l}\text { No } \\
\text { aplica }\end{array}$ & No aplica & $\begin{array}{l}\text { No } \\
\text { aplica }\end{array}$ & No aplica \\
\hline \multirow{2}{*}{$\begin{array}{l}\text { Medio } \\
\text { ambiente }\end{array}$} & 0.289 & 0.554 & 0.589 & 0.132 & 0.390 & 0.261 \\
\hline & $\mathrm{N}=15$ & $\mathrm{~N}=10$ & $\mathrm{~N}=5$ & $\mathrm{~N}=15$ & $\mathrm{~N}=10$ & $\mathrm{~N}=5$ \\
\hline \multirow{2}{*}{$\begin{array}{l}\text { Desarrollo } \\
\text { sustentable }\end{array}$} & -0.157 & 0.582 & -0.923 & 0.501 & 0.692 & 0.134 \\
\hline & $N=7$ & $N=4$ & $N=3$ & $N=7$ & $N=4$ & $N=3$ \\
\hline \multirow{2}{*}{ Climatología } & -0.248 & -0.041 & $-1.0 * *$ & -0.473 & -0.401 & $-1.0 * *$ \\
\hline & $N=6$ & $N=4$ & $N=2$ & $N=6$ & $N=4$ & $N=2$ \\
\hline \multirow{2}{*}{$\begin{array}{l}\text { Tecnología } \\
\text { del medio } \\
\text { ambiente }\end{array}$} & 0.260 & 0.313 & $0.693 *$ & -0.227 & -1.0 & -0.083 \\
\hline & $N=40$ & $N=31$ & $N=9$ & $N=40$ & $N=31$ & $N=9$ \\
\hline \multirow{2}{*}{ Oceanografía } & 0.353 & 0.261 & $-1.0 * *$ & -0.012 & 0.009 & $-1.0 * *$ \\
\hline & $N=10$ & $N=8$ & $N=2$ & $N=10$ & $N=8$ & $N=2$ \\
\hline \multirow{2}{*}{$\begin{array}{l}\text { Otras } \\
\text { especialidades }\end{array}$} & $0.744^{*}$ & $0.792 *$ & No & 0.139 & 0.269 & No aplica \\
\hline & $N=9$ & $N=8$ & $N=1$ & $N=9$ & $N=8$ & $N=1$ \\
\hline
\end{tabular}

Notas: ${ }^{1}$ Ecología es la única disciplina con el investigador emérito. N: Número de investigadores. *: La correlación es significativa en el nivel 0.05 ( 2 colas). **: La correlación es significativa en el nivel 0.01 (2 colas).

Fuente: Elaboración propia

De esta misma forma, considerando a todas las disciplinas ambientalistas en conjunto pero desagregando los niveles de los investigadores nacionales, es que se distingue alguna correlación entre alguna de las métricas de centralidad y el índice de impacto de los científicos. Ejemplo de ello es la correlación baja de $0.322 * *$ aunque significativa en el nivel 0.01 (dos colas) entre el grado y el impacto de las publicaciones de los 65 científicos de nivel dos. Esto indica que el número de coautores inciden débilmente en el impacto de sus 


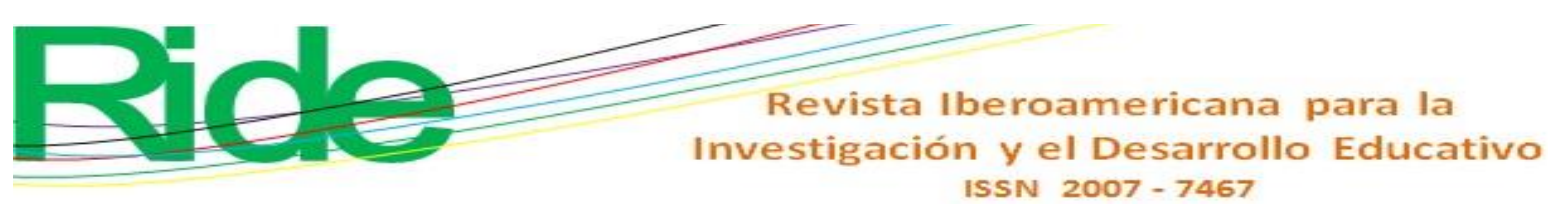

artículos. Mientras que para los 22 investigadores nacionales nivel tres existe una correlación inversa moderada, con un valor de -0.558 y significativa en el nivel 0.01 (dos colas) entre la cercanía y el índice $h$, lo que se traduce en que a mayor independencia (cercanía) de los científicos menor es el impacto de sus publicaciones.

En cuanto a las disciplinas, particularmente los investigadores de nivel tres pertenecientes a tecnología de medio ambiente son quienes correlacionan en forma alta con un valor de Pearson de 0.693 su centralidad de grado con el impacto de sus artículos publicados. De igual manera, los científicos de nivel dos de otras especialidades con una correlación alta de 0.792 , ambas correlaciones significativas en el nivel 0.05 ( 2 colas). En los casos de oceanografía y climatología, se observa una correlación inversa muy alta y significativa para los investigadores de nivel tres, entre la centralidad de grado y de cercanía con el índice de impacto de los investigadores, lo que en gran medida se explica porque en ambos casos solo son dos investigadores los que forman parte de estas categorías.

\section{Conclusiones}

México como uno de los países emergentes se encuentra en el proceso de desarrollar sistemas de investigación científica competitivos. Ante ello es esencial identificar sus fortalezas de investigación y a las redes de colaboración para mejorar la visibilidad e impacto de sus científicos. Una de estas redes es la de la coautoría de los científicos ambientalistas. En dicha red se identifican a 88 investigadores nacionales de los más distinguidos: 1 emérito, 22 en nivel dos y 65 en nivel tres, pertenecientes a 7 campos disciplinarios. Es la disciplina de tecnología de medio ambiente la que concentra el mayor número de investigadores nacionales con $45 \%$ del total de ellos; mientras que en ecología es donde se encuentra el único científico emérito. Desarrollo sustentable es la disciplina que, con $8 \%$ de los investigadores totales, más produce publicaciones con $52 \%$.

Una primera conclusión que se deriva de este estudio es que los investigadores ambientalistas publican en forma colaborativa: $97 \%$ de los artículos son escritos en coautoría; además, de que todos los científicos con una centralidad de grado promedio de 14 se encuentran bien conectados. En este sentido, los autores con grados más altos se convierten, por su posición central en la red, en los más influyentes, los cuales se encuentran en desarrollo sustentable y medio ambiente. No obstante, la red presenta un bajo promedio de centralidad de cercanía con un coeficiente de 0.215; es ecología con 0.569 la disciplina 


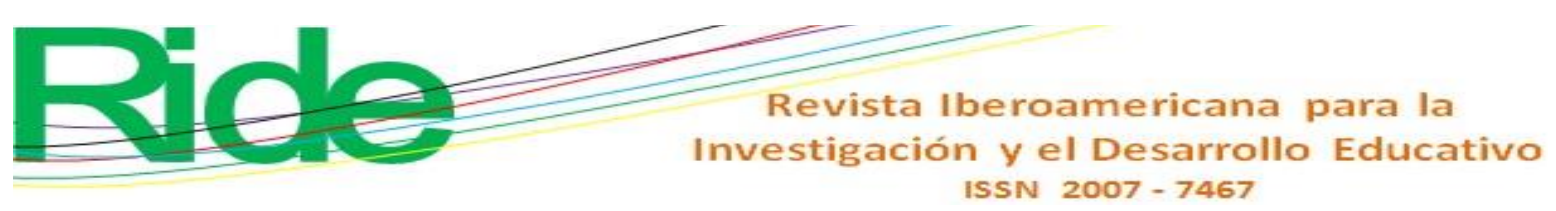

con el mayor valor de cercanía. De igual forma, climatología y desarrollo sustentable cuentan con coeficientes por arriba de 0.5 . Esto indica que sus investigadores se encuentran más cerca de los demás investigadores de la red de coautoría, por lo que en ellas están los científicos más centrales, mismos que pueden evitar el potencial control e influencia de los otros. Lo que se reafirma en ecología por tener un solo investigador nacional: es la disciplina más centralizada de todas.

Finalmente, se concluye con la inexistente correlación entre la centralidad de grado y la centralidad de cercanía con el índice de impacto de los científicos ambientalistas eméritos y niveles dos y tres de México. La única disciplina que evidencia correlación entre la centralidad de grado y el impacto de las obras publicadas de sus investigadores es la catalogada como otras especialidades, que presenta un alto y significativo coeficiente de Pearson de 0.744. Es decir, el número de coautores inciden fuertemente en el impacto de sus artículos. De igual forma, entre el grado y el impacto de las publicaciones existe una correlación baja de 0.322 aunque significativa de los 65 científicos de nivel dos de todas las disciplinas. Ahora bien, para los 22 investigadores nacionales nivel tres existe una correlación inversa moderada, con un valor de -0.558 , y significativa entre la cercanía y el índice $h$, lo que se traduce en que a mayor independencia (cercanía) de los científicos menor es el impacto de sus publicaciones.

Los estudios generales en la literatura especializada en este tema evidencian empíricamente que las colaboraciones contribuyen a la visibilidad y productividad científica. No obstante, a la luz de los resultados obtenidos en esta investigación, se recomienda para futuros estudios de las redes de coautoría de los científicos mexicanos confirmar o no la incidencia de las métricas de centralidad en el impacto de sus respectivas publicaciones. 


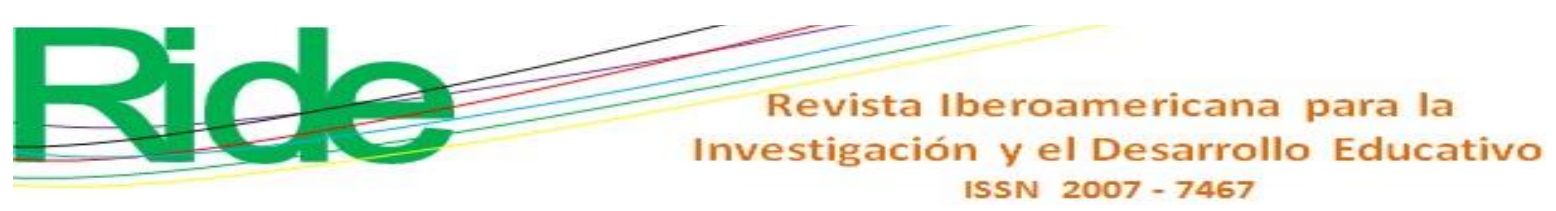

\section{Referencias}

Badar, K., Hite, J. M. and Badir, Y. F. (2014). The moderating roles of academic age and institutional sector on the relationship between co-authorship network centrality and academic research performance. Aslib Journal of Information Management, 66(1), 3853. Retrieved from https://doi.org/10.1108/AJIM-05-2013-0040.

Badar, K., Hite, J. M. and Yuosre, Y. F. (2013). Examining the relationship of co-authorship network centrality and gender on academic research performance: the case of chemistry researchers in Pakistan. Scientometrics, 94(2), 755-775. Retrieved from https://doi.org/10.1007/s11192-012-0764-z.

Barabasi, A. L. and Albert, R. (1999). Emergence of scaling in random networks. Science, 286(5439), 509-512.

Batista, P. D., Campitel, M. G., Kinouchi, O. and Martinez, A. S. (2006). Is it possible to compare researchers with different scientific interests?. Scientometrics, 68(1), 179189.

Bordons, M., Aparicio, J., González-Albo, B. and Díaz-Faes, A. A. (2015). The relationship between the research performance of scientists and their position in co-authorship networks in three fields. Journal of Informetrics, 9(1), 135-144. Retrieved from https://doi.org/10.1016/j.joi.2014.12.001.

Bornmann, L., Mutz, R., Neuhaus, C. and Daniel, H. (2008). Citation counts for research evaluation: standards of good practice for analyzing bibliometric data and presenting and interpreting results. Ethics in Science and Environmental Politics, 8(1), 93-102.

Bridgstock, M. (1991). The quality of single and multiple authored papers; An unresolved problem. Scientometrics, 21(1). 37-48. Retrieved from https://doi.org/10.1007/BF02019181.

Costas, R. and Bordons, M. (2007). The h-index: advantages, limitations and its relation with other bibliometric indicators at the micro-level. Journal of Informetrics, 1(3), 193-203. Retrieved from https://doi.org/10.1016/j.joi.2007.02.001.

De Stefano, D., Fuccella, V., Prosperina, M. and Zaccarin, S. (2013). The use of different data sources in the analysis of co-authorship networks and scientific performance. Social Networks, 35(3), 370-381.

Dill, D. D. (2009). Convergence and diversity: the role and influence of university rankings. In Kehm, B.M. and Stensaker, B. (eds.), University Rankings, Diversity, and the New 


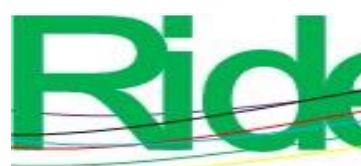

Revista Iberoamericana para la Investigación y el Desarrollo Educativo ISSN $2007-7467$

Landscape of Higher Education, Sense Publishers, (pp. 97-116). Rotterdam, The Netherlands: Sense Publishers.

Dill, D. D., and Soo, M. (2005). Academic quality, league tables, and public policy: a crossnational analysis of university ranking systems, Higher Education, 49(4), 495-533. Retrieved from https://doi.org/10.1007/s10734-004-1746-8.

Eaton, J. P., Ward, J. C., Kumar, A. and Reingen, P. H. (1999). Structural analysis of coauthor relationships and author productivity in selected outlets for consumer behavior research. Journal of Consumer Psychology, 8(1), 39-59. Retrieved from https://doi.org/10.1207/s15327663jcp0801_02.

Egghe, L. (2006). Theory and practise of the g-index. Scientometrics, 69(1),131-52. Retrieved from https://doi.org/10.1007/s11192-006-0144-7.

Fischbach, K., Putzke, J. and Schoder, D. (2011). Co-authorship networks in electronic markets research. Electronic Markets, 21(1), 19-40. Retrieved from https://doi.org/10.1007/s12525-011-0051-5.

Freeman, L. C. (1979). Centrality in networks: I. conceptual clarification. Social Networks, 1(3), 215-239. Retrieved from https://doi.org/10.1016/0378-8733(78)90021-7.

Gazni, A., Sugimoto, C. R. and Didegah, F. (2012). Mapping world scientific collaboration: Authors, institutions, and countries. Journal of the American Society for Information Science and Technology, 63(2), 323-335.

González-Brambila, C. N. (2014). Social capital in academia. Scientometrics, 101(3), 16091625. Retrieved from https://doi.org/10.1007/s11192-014-1424-2.

Hirsch, J. E. (2005). An index to quantify an individual's scientific research output. Proceedings of the National Academy of Sciences, 102(46), 16569-16572. Retrieved from https://doi.org/10.1073/pnas.0507655102.

Katz, J. and Hicks, D. (1997). How much is a collaboration worth? A calibrated bibliometric model. Scientometrics, 40(3), 541-554. Retrieved from https://doi.org/10.1007/BF02459299.

Kelly, C. D. and Jennions, M. D. (2006). The h-index and career assessment by numbers. Trends in Ecology and Evolution, 21(4), 167-170. Retrieved from https://doi.org/10.1016/j.tree.2006.01.005. 


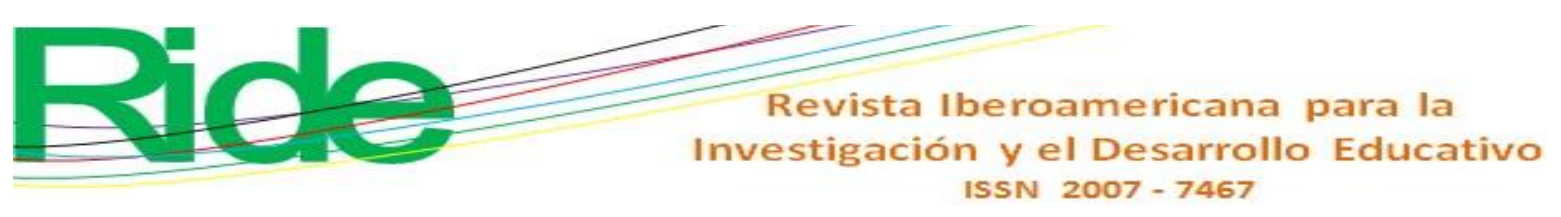

Kronegger, L., Ferligoj, A. and Doreian, P. (2011). On the dynamics of national scientific systems. Quality \& Quantity 45(5), 989-1015. Retrieved from https://doi.org/10.1007/s11135-011-9484-3.

Kumar, S and Mohd. J. (2014). Relationship between authors' structural position in the collaboration network and research productivity: Case of Indian earth scientists. Program, 48(4), 355-369. Retrieved from https://doi.org/10.1108/PROG-01-20130002.

Lee, D. H., Seo, I. W. and Choe, H.C. (2012). Collaboration network patterns and research performance: the case of Korean public research institutions. Scientometrics, 91(3), 925-942. Retrieved from https://doi.org/10.1007/s11192-0110602-8.

Lee, S. and Bozeman, B. (2005). The impact of research collaboration on scientific productivity. Social Studies of Science, 35(5), 673-702. Retrieved from https://doi.org/10.1177/0306312705052359.

Li, E. Y., Liao, C. H. and Yen, R. (2013). Co-authorship networks and research impact: a social capital perspective. Research Policy, 42(9), 1515-1530. Retrieved from https://doi.org/10.1016/j.respol.2013.06.012.

Liao, C. H. (2011). How to improve research quality? Examining the impacts of collaboration intensity and member diversity in collaboration networks. Scientometrics, 86(3), 741761. Retrieved from https://doi.org/10.1007/s11192-010-0309-2.

Liberman, S. and Wolf, K. B. (2013). Scientific communication in the process to coauthor ship. In Feist, G. y Gorman, M. (eds.), Handbook of Psychology of Science (pp. 123147). New York, United States: Springer publishing company.

Lopaciuk-Gonczaryk, B. (2016). Collaboration strategies for publishing articles in international journals - A study of Polish scientists in economics. Social Networks, (44), 50-63. Retrieved from https://doi.org/10.1016/j.socnet.2015.07.001.

Lotka, A. (1926). The Frequency distribution of scientific productivity. Journal of the Washington Academy of Sciences, 16(12), 317-323.

McFadyen, A. M. and Cannella, J. A. (2017). Social capital and knowledge creation: diminishing returns of the number and strength of exchange relationships. Academy of Management Journal, 47(5), 735-746. Retrieved from https://doi.org/10.5465/20159615. 


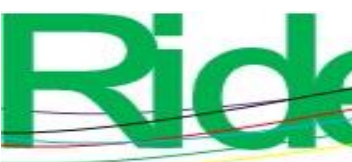

Revista Iberoamericana para la Investigación y el Desarrollo Educativo ISSN $2007-7467$

Munoz, D. A., Queupil, J. P. and Fraser, P. (2016). Assessing collaboration networks in educational research. International Journal of Educational Management, 30(3), 416436. Retrieved from http://dx.doi.org/10.1108/IJEM-11-2014-0154.

Newman, M. E. (2001a). Scientific collaboration networks. I. Network construction and fundamental results. Physical Review E, 64(1). Retrieved from https://doi.org/10.1103/PhysRevE.64.016131.

Newman, M. E. (2001b). Scientific collaboration networks. II. Shortest paths, weighted networks, and centrality. Physical Review E, 64(1). Retrieved from https://doi.org/10.1103/PhysRevE.64.016132.

Newman, M. E. (2004a). Coauthorship networks and patterns of scientific collaboration. Proceedings of the National Academy of Sciences, 101(1), 5200-5205. Retrieved from https://doi.org/10.1073/pnas.0307545100.

Newman, M. E. (2004b). Who Is the Best Connected Scientist? A Study of Scientific Coauthorship Networks. In Ben-Naim, E., Frauenfelder, H. and Toroczkai, Z. (eds.), Complex Networks. Berlin, Germany: Springer.

Nieminen, J. (1974). On centrality in a graph. Scandinavian Journal of Psychology 15:322336. DOI: 10.1111/j.1467-9450.1974.tb00598.x

Peters, R. H. (1991). A Critique for Ecology. United Kingdom: Cambridge University Press.

Pike, T. W. (2010). Collaboration networks and scientific impact among behavioral ecologists. Behavioral Ecology, 21(2), 431-435. Retrieved from https://doi.org/10.1093/beheco/arp194.

Ponomariov, B. L. and Boardman, P. C. (2010). Influencing scientist's collaboration and productivity patterns through new institutions: University research centers and scientific and technical human capital. Research Policy, 39(5), 613-624. Retrieved from https://doi.org/10.1016/j.respol.2018.03.008.

Sidiropoulos, A., Katsaros, D. and Manolopoulos, Y. (2007). Generalized h-index for disclosing latent facts in citation networks. Scientometrics, 72(2), 253-280. Retrieved from https://doi.org/10.1007/s11192-007-1722-z.

Sooryamoorthy, R. (2009). Do Types of Collaboration Change Citation? Collaboration and Citation Patterns of South African Science Publications. Scientometrics, (81). Retrieved from https://doi.org/10.1007/s11192-009-2126-z. 


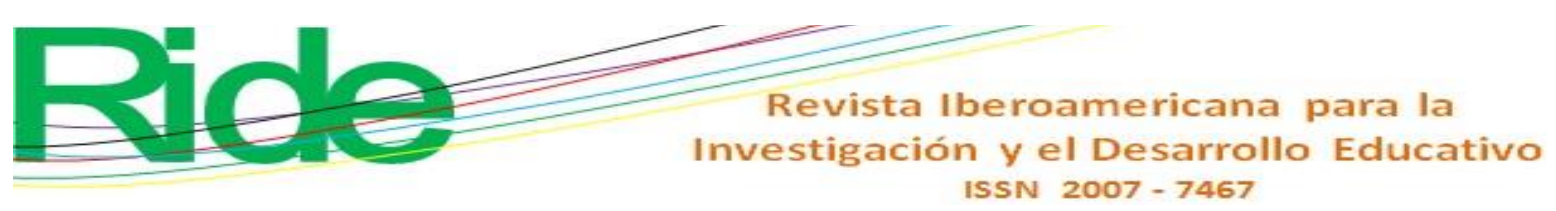

Van Raan, A. F. J. (2006). Comparisons of the Hirsch-index with standard bibliometric indicators and with peer judgment for 147 chemistry research groups. Scientometrics, 67(3), 491-502. Retrieved from https://doi.org/10.1556/Scient.67.2006.3.10.

Yu Cheng, M., Wah Hen, K., Piew Tan, H. and Fai Fok, K. (2013). Patterns of co-authorship and research collaboration in Malaysia. Aslib Proceedings: New Information Perspectives, 65(6), 659-674. Retrieved from https://doi.org/10.1108/AP-12-20120094.

Zuckerman, H. (1967). Nobel laureates in science: Patterns of productivity, collaboration, and authorship. American Sociological Review, 32(3), 391-403. Retrieved from http://dx.doi.org/10.2307/2091086. 
Revista Iberoamericana para la Investigación y el Desarrollo Educativo ISSN $2007-7467$

\section{Anexo}

Tabla 3. Correlaciones por disciplina ambientalista: métricas de centralidad e índice $h$

\begin{tabular}{|c|c|c|c|c|c|c|c|}
\hline Id & $\begin{array}{l}\text { Nivel } \\
\text { SNI }\end{array}$ & Disciplina & Grado & Cercanía & Índice $(h)$ & $\begin{array}{c}\text { Correlación } \\
\text { Grado - } h\end{array}$ & $\begin{array}{c}\text { Correlación } \\
\text { Cercanía - } \\
h\end{array}$ \\
\hline Id 1 & Emérito & Ecología & 13.17 & 0.5829 & 29 & No aplica & No aplica \\
\hline Id 2 & 3 & \multirow{15}{*}{$\begin{array}{c}\text { Medio } \\
\text { ambiente }\end{array}$} & 11.57 & 0.1874 & 21 & \multirow{15}{*}{0.289} & \multirow{15}{*}{0.132} \\
\hline Id 3 & 3 & & 10.35 & 0.1394 & 26 & & \\
\hline Id 4 & 2 & & 14.34 & 0.1636 & 14 & & \\
\hline Id 5 & 3 & & 10.35 & 0.1289 & 18 & & \\
\hline Id 6 & 3 & & 9.476 & 0.141 & 13 & & \\
\hline Id 7 & 2 & & 9.54 & 0.1319 & 11 & & \\
\hline Id 8 & 2 & & 12.64 & 0.1479 & 14 & & \\
\hline Id 9 & 2 & & 41.6 & 0.1678 & 24 & & \\
\hline Id 10 & 3 & & 15.39 & 0.1608 & 25 & & \\
\hline Id 11 & 2 & & 10.36 & 0.1445 & 13 & & \\
\hline Id 12 & 2 & & 13.54 & 0.1222 & 2 & & \\
\hline Id 13 & 2 & & 13.81 & 0.1643 & 11 & & \\
\hline Id 14 & 2 & & 11.23 & 0.5729 & 18 & & \\
\hline Id 15 & 2 & & 18.87 & 0.138 & 12 & & \\
\hline Id 16 & 2 & & 7.63 & 0.5434 & 16 & & \\
\hline Id 17 & 3 & \multirow{7}{*}{$\begin{array}{l}\text { Desarrollo } \\
\text { sustentable }\end{array}$} & 4.4 & 0.6666 & 11 & \multirow{7}{*}{-0157} & \multirow{7}{*}{0.501} \\
\hline Id 18 & 3 & & 44.09 & 0.6549 & 6 & & \\
\hline Id 19 & 3 & & 14.32 & 0.1503 & 8 & & \\
\hline Id 20 & 2 & & 8.18 & 0.1191 & 4 & & \\
\hline Id 21 & 2 & & 13.75 & 0.1429 & 4 & & \\
\hline Id 22 & 2 & & 12.9 & 0.1039 & 2 & & \\
\hline Id 23 & 2 & & 32.6 & 0.7398 & 5 & & \\
\hline Id 24 & 3 & \multirow{6}{*}{ Climatología } & 17.91 & 0.531 & 4 & \multirow{6}{*}{-0.248} & \multirow{6}{*}{-0.473} \\
\hline Id 25 & 2 & & 6.85 & 0.0921 & 11 & & \\
\hline Id 26 & 3 & & 11.46 & 0.1332 & 18 & & \\
\hline Id 27 & 2 & & 10.49 & 0.1149 & 1 & & \\
\hline Id 28 & 2 & & 15.05 & 0.373 & 3 & & \\
\hline Id 29 & 2 & & 17.02 & 0.1577 & 11 & & \\
\hline Id 30 & 2 & \multirow{11}{*}{$\begin{array}{l}\text { Tecnología } \\
\text { del medio } \\
\text { ambiente }\end{array}$} & 10.35 & 0.62 & 9 & \multirow{11}{*}{0.26} & \multirow{11}{*}{-0.227} \\
\hline Id 31 & 3 & & 10.9 & 0.1502 & 19 & & \\
\hline Id 32 & 3 & & 9.86 & 0.1262 & 17 & & \\
\hline Id 33 & 2 & & 13.21 & 0.128 & 9 & & \\
\hline Id 34 & 3 & & 37.81 & 0.1621 & 24 & & \\
\hline Id 35 & 3 & & 9.15 & 0.1302 & 19 & & \\
\hline Id 36 & 3 & & 10.31 & 0.1221 & 22 & & \\
\hline Id 37 & 2 & & 8.9 & 0.555 & 9 & & \\
\hline Id 38 & 2 & & 18.29 & 0.1369 & 11 & & \\
\hline Id 39 & 3 & & 11.38 & 0.1496 & 17 & & \\
\hline Id 40 & 3 & & 11.07 & 0.1688 & 19 & & \\
\hline
\end{tabular}


Revista lberoamericana para la

Investigación y el Desarrollo Educativo

\begin{tabular}{|c|c|c|c|c|c|c|c|}
\hline Id & $\begin{array}{l}\text { Nivel } \\
\text { SNI }\end{array}$ & Disciplina & Grado & Cercanía & Índice $(h)$ & $\begin{array}{c}\text { Correlación } \\
\text { Grado - } h\end{array}$ & $\begin{array}{c}\text { Correlación } \\
\text { Cercanía - } \\
h\end{array}$ \\
\hline Id 41 & 2 & & 11.48 & 0.1388 & 9 & & \\
\hline Id 42 & 2 & & 16.45 & 0.1681 & 6 & & \\
\hline Id 43 & 2 & & 12.35 & 0.187 & 19 & & \\
\hline Id 44 & 2 & & 10.73 & 0.1176 & 2 & & \\
\hline Id 45 & 3 & & 15.23 & 0.1611 & 19 & & \\
\hline Id 46 & 2 & & 12.97 & 0.1823 & 10 & & \\
\hline Id 47 & 2 & & 16.86 & 0.1516 & 15 & & \\
\hline Id 48 & 2 & & 8.58 & 0.1334 & 7 & & \\
\hline Id 49 & 2 & & 18.88 & 0.1616 & 6 & & \\
\hline Id 50 & 2 & & 18.97 & 0.1472 & 16 & & \\
\hline Id 51 & 2 & & 18.86 & 0.1455 & 13 & & \\
\hline Id 52 & 3 & & 14.77 & 0.1785 & 17 & & \\
\hline Id 53 & 2 & & 8.51 & 0.5626 & 11 & & \\
\hline Id 54 & 2 & & 16.44 & 0.1641 & 3 & & \\
\hline Id 55 & 2 & & 11.7 & 0.5627 & 13 & & \\
\hline Id 56 & 2 & & 14.74 & 0.5532 & 12 & & \\
\hline Id 57 & 2 & & 15.78 & 0.1763 & 23 & & \\
\hline Id 58 & 2 & & 26.1 & 0.1766 & 16 & & \\
\hline Id 59 & 2 & & 17.98 & 0.1626 & 16 & & \\
\hline Id 60 & 2 & & 9.61 & 0.5902 & 7 & & \\
\hline Id 61 & 2 & & 14.75 & 0.1239 & 9 & & \\
\hline Id 62 & 2 & & 13.29 & 0.6112 & 11 & & \\
\hline Id 63 & 2 & & 9.18 & 0.1774 & 10 & & \\
\hline Id 64 & 2 & & 18.88 & 0.1432 & 6 & & \\
\hline Id 65 & 2 & & 18.2 & 0.1619 & 12 & & \\
\hline Id 66 & 2 & & 13.9 & 0.1196 & 17 & & \\
\hline Id 67 & 2 & & 12 & 0.1436 & 12 & & \\
\hline Id 68 & 2 & & 18.89 & 0.1697 & 20 & & \\
\hline Id 69 & 2 & & 14.78 & 0.1523 & 16 & & \\
\hline Id 70 & 3 & & 9.7 & 0.5547 & 5 & & \\
\hline Id 71 & 2 & & 14.25 & 0.1477 & 11 & & \\
\hline Id 72 & 2 & & 10.18 & 0.6412 & 3 & & \\
\hline Id 73 & 2 & & 15.53 & 0.6987 & 9 & & \\
\hline Id 74 & 3 & & 9.51 & 0.1337 & 6 & & \\
\hline Id 75 & 2 & & 7 & 0.3962 & 13 & & \\
\hline Id 76 & 2 & & 14.03 & 0.5472 & 17 & & \\
\hline Id 77 & 2 & & 7.57 & 0.1285 & 8 & & \\
\hline Id 78 & 2 & & 16.82 & 0.1145 & 11 & & \\
\hline Id 79 & 2 & & 10.91 & 0.1158 & 6 & & \\
\hline Id 80 & 2 & \multirow{5}{*}{$\begin{array}{c}\text { Otras } \\
\text { especialidades }\end{array}$} & 13.15 & 0.1451 & 10 & \multirow{5}{*}{$0.744^{*}$} & \multirow{5}{*}{0.139} \\
\hline Id 81 & 3 & & 12.8 & 0.1533 & 3 & & \\
\hline Id 82 & 2 & & 10.23 & 0.162 & 7 & & \\
\hline Id 83 & 2 & & 11.87 & 0.1368 & 6 & & \\
\hline Id 84 & 2 & & 20.18 & 0.1541 & 15 & & \\
\hline
\end{tabular}



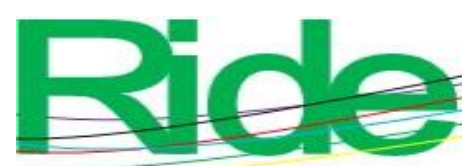

Revista Iberoamericana para la Investigación y el Desarrollo Educativo

\begin{tabular}{|c|c|c|c|c|c|c|c|}
\hline Id & $\begin{array}{c}\text { Nivel } \\
\text { SNI }\end{array}$ & Disciplina & Grado & Cercanía & Índice $(h)$ & $\begin{array}{c}\text { Correlación } \\
\text { Grado - } h\end{array}$ & $\begin{array}{c}\text { Correlación } \\
\text { Cercanía - } \\
\boldsymbol{h}\end{array}$ \\
\hline Id 85 & 2 & & 17.1 & 0.177 & 15 & & \\
\hline Id 86 & 2 & & 15.3 & 0.0914 & 12 & & \\
\hline Id 87 & 2 & & 13 & 0.123 & 9 & & \\
\hline Id 88 & 2 & & 15.32 & 0.156 & 18 & & \\
\hline
\end{tabular}

Nota: *: La correlación es significativa en el nivel 0.05 (2 colas).

Fuente: Elaboración propia 